\title{
Os Usos de Simuladores Durante a Formação Profissional para a Condução de Transportes Rodoviários
}

\author{
Thiago Drumond Moraes \\ Universidade Federal do Espírito Santo, ES, Brasil. \\ Paulo César Zambroni-de-Souza \\ Universidade Federal da Paraíba, PB, Brasil. \\ Yves Schwartz \\ Université d'Aix-Marseille, França.
}

\begin{abstract}
Resumo: Este estudo teórico pretende contribuir com o debate sobre o uso de simuladores nos processos de formação para os profissionais de transporte terrestre rodoviário. Nesse tipo de transporte no Brasil ocorrem muitos acidentes, configurando-se em um problema de saúde pública. Procurou-se analisar criticamente, a partir da produção acadêmica sobre uso de simuladores, suas potencialidades em situações de formação para condutores profissionais. A partir de uma perspectiva ergológica, que permite a articulação com outras clínicas do trabalho, também utilizadas, o artigo tem como objetivos: discutir as características que conformam a atividade de condução, fazer uma análise dos pressupostos epistemológicos subjacentes às pesquisas e treinamentos que usam simuladores e articular criticamente tais pontos para avaliar a pertinência do uso de simuladores em processos de formação profissional. Evidenciou-se que os processos de formação profissional mediado pelo uso de simuladores podem ser pertinentes para a grave situação do trânsito brasileiro, embora se reconheça ausência de pesquisas nacionais sobre a temática, bem como alguns possíveis limites da perspectiva epistemológica utilizadas nas pesquisas internacionais, afetando a eficácia deste uso.
\end{abstract}

Palavras-chave: Simulação, Transportes, Formação Profissional, Acidentes de Trânsito.

\section{Usages of Simulators During Professional Training for Driving road Transportation}

\begin{abstract}
This theoretical study aims to contribute to the debate on the usagee of simulators in the training processes of road land transportation professionals. Many accidents occur in this type of transportation in Brazil, constituting a public health problem. Simulators usage and its potentialities were critically analyzed, from the academic literature on simulators usage, in professional driving training situations. From an ergological perspective, which allows the articulation with the approach of others clinics of work, that were also used, the article aims to describe the characteristics that mold the driving activity, to analyze the epistemological assumptions underlying the research and training using simulators, and to critically articulate these points to assess the appropriateness of simulators usage in training processes. It was highlighted that the professional training processes mediated by the use of simulators may be relevant to the serious situation of Brazilian traffic, although the absence of national research on this topic is evident, as well as some possible limits of epistemological perspective used in international surveys, affecting the effectiveness of simulators usage.
\end{abstract}

Keywords: Simulation, Transportation, Professional Education, Motor Traffic Accidents. 


\title{
Usos de Simuladores Durante la Formación Profesional para la Conducción de Vehículos de Transporte Terrestre por Carretera
}

\begin{abstract}
Resumen: Este estudio teórico pretende contribuir al debate sobre el uso de simuladores en los procesos de formación para los profesionales del transporte terrestre por carretera. Ocurren muchos accidentes en este tipo de transporte en Brasil, y esto representa un problema de salud pública. Se buscó analizar críticamente, desde la literatura académica sobre el uso de simuladores, sus potencialidades en situaciones de formación de conductores profesionales. Desde una perspectiva ergológica, que permite la articulación con otras clínicas del trabajo, también utilizadas, el artículo tiene como objetivo abordar las características que conforman la actividad de conducción, hacer un análisis de los presupuestos epistemológicos subyacentes a la investigación y formación que utilizan simuladores, y articular críticamente esos puntos para evaluar la pertinencia del uso de simuladores en los procesos de formación. Se mostró que los procesos de formación mediados por el uso de simuladores pueden ser relevantes para la grave situación de tránsito brasileño, mientras se reconoce la ausencia de una investigación nacional sobre el tema, así como algunos posibles límites de la perspectiva epistemológica utilizada en la investigación internacional, afectando la eficacia de este uso.
\end{abstract}

Palabras clave: Simulación, Transportes, Formación Profesional, Accidentes de Tránsito.

\section{Introdução}

Esse texto tem como objetivo refletir criticamente sobre o alcance dos usos de simuladores de direção para a formação profissional de condutores dos transportes rodoviários terrestres, por meio de análises sobre o modo como estes equipamentos vêm sendo utilizados em pesquisas e processos de formação de trabalhadores deste setor.

No caso específico da Psicologia do Trabalho e Organizacional (PTO), a formação profissional é tema central desde seu surgimento (Sampaio, 1998; Spector, 2002). Se nos moldes tayloristas, a formação se limitava a treinamento e adestramento de operações repetidas, nas últimas décadas a competência e o reconhecimento da complexidade que o trabalho aporta (Schwartz, 1998; Schwartz, \& Durrive, 2010) ganham importância cada vez maior, de tal forma que é necessário levar em conta os ingredientes presentes em cada situação profissional, em especial no caso de condutores, como esse artigo procura abordar. Há, neste domínio, um vasto campo a ser desenvolvido que pode permitir avançar a PTO e a segurança de trabalhadores e de usuários comuns das vias terrestres.

Estas ponderações fazem-se necessárias já que é recente no país a autorização do uso dessa ferramenta em formações de condutores de veículos leves - carteiras A e B (Brasil, 2014;2015). Lança-se mão desse instrumento como uma das estratégias delineadas pelo Conselho Nacional do Trânsito (Contran) para enfrentar o grave quadro de acidentes de trânsito no Brasil, haja vista a alta incidência de óbitos em colisões e atropelamentos constatada a partir do Sistema Nacional de Informação de Mortalidade (SIM) (Bacchieri, \& Barros, 2011). Mesmo considerando-se imprecisões nas informações brasileiras sobre os acidentes de trabalho neste setor, se reconhece neste campo índices de mortalidade maiores que em países desenvolvidos (Bacchieri, \& Barros, 2011) e em outros setores profissionais (Santana, Nobre, \& Waldvogel, 2005). Requer-se, portanto, políticas específicas de prevenção de acidentes de trabalho para o setor.

Em contrapartida, a despeito das exigências do Contran para que os Centros de Formação de Condutores adquiram simuladores e os autorizem a utilizá-los em aulas de condução como etapa para o ensino de direção aos alunos, raras são as pesquisas nacionais justificando esse amplo uso dos simuladores (Lucas et al., 2013) ou que avaliam seus efeitos em processos de formação (Mendes, Mendes, \& Bomfin, 2013), sendo ainda mais raros os que investigam o uso desses instrumentos em escala nacional. Essa escassez vale também para as formações profissionais, muito embora o uso de simuladores de condução para este fim - carteiras C, D e E - ainda não tenha sido regu- 
lamentado no país, diferentemente do que ocorre na Comunidade Europeia (European Parliament, 2003).

Verificamos que em países europeus, nos quais o índice de mortalidade em transportes terrestres vem caindo nos últimos 10 anos, o interesse pela formação profissional é estratégico (World Health Organization, 2013). No Brasil, o cenário parece ser um pouco diferente, já que treinamentos são realizados aparentemente sem as devidas avaliações mais criteriosas e sem aparente embasamento científico, uma vez que excepcionalmente são encontradas pesquisas nacionais sobre formação profissional de trabalhadores dos transportes rodoviários. Entretanto, pesquisas demonstram que a despeito da qualidade da formação, há uma contínua partilha coletiva de saberes da/na atividade, ocorrendo nas conversas cotidianas, assemelhando-se a um tipo de treinamento informal pelos pares (Diniz, Assunção, \& Lima, 2005; Moraes, 2008).

A despeito da carência de pesquisas nacionais, há indícios de que o uso de simuladores de direção veicular - doravante simuladores de condução - em situações de formação enseja redução de acidentes (Allen, Park, Cook, \& Fiorentino, 2007), sobretudo por meio da aprendizagem e aprimoramento de habilidades que, em situações reais, seriam muito arriscadas a serem desenvolvidas nos novatos. Por outro lado, temos razão para supor que as pesquisas internacionais sobre formação profissional por simuladores negligenciam alguns aspectos cruciais em seus desenhos metodológicos e epistemológicos, tais como investigação de pequena quantidade e variabilidade de competências simuladas pelos instrumentos e a falta de referências teóricas claras que orientem os treinamentos (Moraes, 2016). Estes e outros aspectos, se forem incluídos nos estudos sobre o tema, poderão ampliar seus escopos, validades e aplicabilidades, sobretudo para formações voltadas para a condução profissional. Diante da escassez de debates nacionais sobre o assunto, realizamos uma análise das pesquisas internacionais sobre formações profissionais que usam simuladores de condução, com o objetivo de examinar seus avanços, limites e desafios, bem como de refletir criticamente sobre os pressupostos teórico-epistemológicos a elas subjacentes, e avaliar a pertinência dos usos de simuladores em formações profissionais. Constatamos que alguns pressupostos epistemológicos que embasam tais pesquisas e formações não reconhecem as especificidades de se conduzir em situações de trabalho, o que pode acarretar em diminuição da eficácia desse instrumento. Apresentamos, enfim, alguns caminhos possíveis que talvez contribuam para melhor uso desse instrumento, na expectativa de abrir um debate acadêmico sobre $\mathrm{o}$ assunto.

Após a apresentação do método da investigação, no tópico seguinte faremos uma análise da utilização do simulador tal como a literatura científica internacional a apresenta. Posteriormente, demonstraremos quais as competências são mobilizadas em trabalho de condução profissional, tomando como referência análises ergonômicas da atividade de condutores. Articularemos criticamente, a partir da Ergologia, tais considerações para avaliarmos a pertinência do uso de simuladores de condução em processos de formação profissional tal qual eles vêm sendo investigados em outros países, bem como sugeriremos alguns caminhos de investigação sobre possíveis usos do instrumento. Concluímos o texto demonstrando a pertinência do uso desse equipamento, sublinhando, porém, a necessidade de fazê-lo a partir de novas perspectivas teóricas.

\section{Método}

Para realizarmos a presente análise, apresentaremos apenas alguns dados extraídos de uma revisão da literatura publicada em outro local (Moraes, 2016). Esta pesquisa foi executada a partir da busca de textos de revistas indexadas, trabalhos apresentados em conferências internacionais e relatórios de pesquisas financiadas por países em que tais simuladores já vêm sendo empregados sistematicamente. Para tanto, consultamos algumas bases de dados, entre as quais: CINAHL with Full Text (EBSCO), Cochrane, Education Resources Information Center - ERIC (ProQuest), Medline Complete (EBSCO), WEBofSCIENCE Social science citation index, PsycArticles (APA), SciELO, Scopus (Elsevier), SAGE Journals Online, Traffic Research Information Services (TRIS), Medline, Education Research Complete, SocINDEX, Psychology and Behavioral Sciences Collection, Lilacs, Ibecs, além de consultas à internet (scholar google) e às referências bibliográficas dos textos selecionados.

Realizamos a busca dos textos no mês de setembro de 2015 a partir dos seguintes termos: Driv* simulator; training-educational; professional-commercial-truck-heavy vehicle-bus-taxi-long-haul. Das 465 publicações encontradas nessas consultas, sele- 
cionamos por meio da leitura dos títulos e/ou resumos 49 textos cujo conteúdo versava sobre formações profissionais por meio de simuladores de condução. Dada a quantidade de textos encontrados, a ausência de pesquisas controladas e, por se tratar de uma área ainda em expansão, o único critério de exclusão aplicado foi a disponibilidade das publicações estarem completas para acesso na internet.

Assim, a revisão realizou-se sobre 46 publicações que foram lidas na íntegra e categorizados em categorias estabelecidas previamente em torno das seguintes questões orientadoras: a) justificativa acerca do uso de simuladores na formação de profissionais; b) competências a serem desenvolvidas, métodos empregados e atuação dos formadores; c) públicos-alvo; d) pressupostos teórico-epistemológicos embasam suas ações.

\section{A análise dos pressupostos epistemológicos subjacentes às pesquisas internacionais $e$ as perspectivas abertas a partir da Ergologia}

A simulação induz pessoas a realizarem atividades semelhantes às que realizariam em situações às quais ela busca se remeter. Para tanto, espera-se eliciar no indivíduo as sensações de presença - experiência subjetiva de perceber-se em determinado ambiente, mesmo que estando fisicamente em outro - e imersão - percepção de estar envolvido e interagindo com um ambiente que fornece estímulo contínuo (Masciocchi, Dark, \& Parkhurst, 2006). Quanto mais presença e imersão a simulação permite, melhor é o desempenho do indivíduo na tarefa simulada e, consequentemente, na aquisição das habilidades no ambiente simulado (Morgan et al., 2011). Os simuladores são instrumentos que eliciam percepções e comportamentos sempre controlados e vêm sendo utilizados nas mais diversas atividades humanas, da aviação à medicina, passando pela pilotagem de centrais nucleares à formação de profissionais que trabalham na manutenção de vinhedos, sendo também utilizados nos campos de pesquisa e no desenvolvimento de produtos e de equipamentos (Blanco, Hickman, Hanowski, \& Morgan, 2011; Pastré, 2005a).

Se no campo da condução de transportes aéreos, em medicina e na indústria a simulação já se possui um elevado grau de aplicação e validação (Boet, Jaffrelot, Naik, Brien, \& Granry, 2014; Pastré, 2005a), em condução de veículos ainda não se obteve esse mesmo estatuto de aplicabilidade e aceitabilidade, embora a partir dos anos 2000 se perceba um aumento do uso desse instrumento (Fisher, Rizzo, Caird, \& Lee, 2011).

O uso dos simuladores de condução se justifica por inúmeras razões: relativo baixo custo, razões éticas, para o desenvolvimento de novos equipamentos na interface homem-máquina, e a grande quantidade de dados que podem ser obtidos a partir de simuladores, que não seriam possíveis em situações reais (Carsten, \& Jamson, 2011). Há indícios de que os simuladores de condução sejam instrumentos capazes de produzir aprendizagem nos usuários e que esses aprendizados são parcialmente transferidos à realidade (Goode, Salmon, \& Lenné, 2013), produzindo efeitos, inclusive, de aumento de segurança no trânsito. Pesquisas demonstram também que os usuários tendem a classificar os simuladores positivamente, de modo a reforçar sua fidelidade visual e psicológica (Brook-Carter et al., 2004). Por outro lado, no que tange aos simuladores para trabalhadores dos transportes terrestres, as evidências são ainda insuficientes, requerendo mais pesquisas sobre o assunto (Goode et al., 2013; Grüneberg, \& Schröder, 2012).

Há validações, em geral qualitativas, da fidelidade psicológica dos simuladores, ou, em outros termos, da percepção de realismo (imersão e presença) que esses instrumentos produziram nos usuários tanto para condutores de ônibus (National Center for Transit Research, 2013), quanto operadores de veículos removedores de neve (Kihl, Herring, Wolf, McVey, \& Kovuru, 2006), e para caminhoneiros (Morgan et al., 2011).

Verificou-se, também, a possibilidade de transferência (transferability) de aprendizagens realizadas por meio desse instrumento (Blanco et al., 2011). Por exemplo, demonstrou-se que o uso de simuladores de condução na formação de condutores profissionais novatos tem uma validade relativa, sobretudo no que tange ao treinamento de habilidades específicas do processo de obtenção da licença (Morgan et al., 2011). Estudos demonstram que seu uso aprimorou outras habilidades procedimentais tais como as manobras para estacionamento dos veículos (Uhr, Felix, Williams, \& Krueger, 2003). Nesta pesquisa, verificou-se que os alunos que utilizaram o simulador para desenvolver suas habilidades de estacionar os caminhões de tipo trailer conseguiam replicar suas aprendizagens em equipamentos não virtuais cometendo apenas um pouco mais de erros que os alunos 
que aprenderam essas manobras em veículos reais. Estes autores sugerem que os erros cometidos em veículos reais pelos alunos que aprenderam no simulador são efeitos de uma transferência negativa de conhecimentos aprendidos no instrumento que, sendo mais difícil de operar, habituava os aprendizes a tomarem mais cautela ao conduzir. Chama-se, assim, atenção à qualidade do desenho dos simuladores para o processo de formação, buscando-se evitar transferências negativas de aprendizagem.

Finalmente, demonstrou-se que os simuladores contribuem também para o desenvolvimento de habilidades mais cognitivas, tais como a busca visual de potenciais riscos no ambiente (Morgan et al., 2011). Neste caso, há indícios que tais aparelhos sirvam como instrumentos para formações continuadas de profissionais qualificados, sobretudo para o enfrentamento de situações raras e perigosas, como condução no gelo, estouro dos pneus e em ambientes de pouca visibilidade. Resultados semelhantes foram obtidos no estudo com condutores de ônibus (Dorn, \& Stannard, 2006). Outra pesquisa sugere que o instrumento contribui para o desenvolvimento de habilidades de percepção e antecipação de situações de risco em condutores de veículos de emergência (Neukum, Lang, \& Krueger, 2003).

Não encontramos estudos com condutores profissionais de outros veículos, tais como motociclistas profissionais, profissão que contribui para o aumento de acidentes de motocicletas em países como o Brasil (Bacchieri, \& Barros, 2011). Ademais, apesar de se justificar o uso de simuladores para a formação profissional para se reduzir o número e gravidade de acidentes e incidentes profissionais, e mesmo que alguns resultados encontrados sugiram essa relação (Kihl et al., 2006; Morgan et al., 2011), não há resultados definitivos que permitam afirmar que o uso desse instrumento na formação profissional reduza acidentes de trabalho (Blanco et al., 2011; Grüneberg, \& Schröder, 2012).

Por outro lado, algumas das pesquisas acima demonstram que após certo tempo a aprendizagem realizada nas formações se perde parcialmente (Strayer, \& Drews, 2003). Outros estudos vêm demonstrando limites ou mesmo questionam o uso de simuladores para produção de conhecimentos aplicáveis em situações reais (Carsten, \& Jamson, 2011), haja vista a defasagem existente entre as capacidades propiciadas pelo ambiente simulado e as condições reais em que os usuários operam (Kihl et al., 2006). Relem- bramos, também, os riscos de transferência negativa de conhecimentos a partir do uso de simuladores com desenvolvimento precário (Uhr et al., 2003).

Desses estudos pode-se depreender um aspecto geral comum a quase todas as pesquisas realizadas com simuladores de condução: baseiam-se em treinamentos de habilidades específicas individuais, geralmente procedimentos sensório-motores gestuais, simples ou complexos, tais como controle dos veículos, troca de marchas, realização de manobras, entre outros. Além disso, o suporte teórico mais citado para a análise e planejamento dos treinamentos e pesquisa é o modelo rasmusseniano do conhecimento organizado hierarquicamente em habilidades técnicas, táticas e estratégicas (Brock, Jacobs, \& Buchter, 2001; Morgan et al., 2011; Van Box Som, 2010; Victor et al., 2011) e sua aplicação no campo da condução a partir do modelo de Michon (Kihl et al., 2006; Van Box Som, 2010). Esses modelos, baseados em três níveis hierárquicos, referem-se aos conhecimentos estratégico (conhecimento), tático (regras) e operacional (habilidade), mobilizados nas ações das pessoas (condutor) em seu meio (trânsito). De modo geral, as habilidades mais procedimentais ( procedural skills), baseadas em domínio sensório-psicomotor-corporal simples, como trocas de marcha, controle do veículo na pista, ou complexas, como estacionamento dos veículos, são mais facilmente replicadas e avaliadas que os conhecimentos mais táticos e mais cognitivos, baseados em regras, como tempo de detecção de perigos ou resposta a eles (Goode et al., 2013). Entretanto, pesquisas sugerem que os conhecimentos mais complexos (high-order cognitives skills) envolvidos na condução, como as tarefas gerais de condução global e geral (tais como responder a situação de risco, obedecer a regras de trânsito e manter o planejamento e tarefas determinadas pelo gerente), também podem ser foco de formações com simuladores, apesar de seu restrito uso para formação de habilidades mais técnicas e específicas (Goode et al., 2013; Mitsopoulos-Rubens, Lenné, \& Salmon, 2013).

Em suma, se há validade parcial dos usos de simuladores em formação profissional, podemos supor que eles vêm sendo utilizados mais frequentemente como instrumento de treinamento corporal para o ofício de condução. Como se o trabalho dos condutores profissionais resumisse-se ao uso do corpo no domínio do automóvel em um meio regulado por regras explícitas que devem ser obedecidas, não importa a condição, 
devendo os condutores estar preparados para, diante de alguma intercorrência inesperada, se comportar de maneira eficaz, segura e dentro dos limites legais. Uma expectativa de um corpo bem treinado, obediente e disciplinado. Um corpo dócil (Foucault, 2009) e cientificamente ideal (Taylor, 2004).

Por outro lado, tomando-se a consideração de Hubault (2011a) de que as características singulares da condução em situação de trabalho requerem dos pesquisadores reconhecerem que essa atividade, para além de atividade de condução, é uma atividade de trabalho, vislumbra-se aqui a necessidade de situar as dimensões da atividade do trabalho para além do que se define pelas prescrições e pela tarefa. Segue-se aqui a linhagem da Ergonomia da Atividade, ou Ergonomia Francófona (Falzon, 2007) e da Ergologia (Schwartz, \& Durrive, 2010). Nessa direção, urge repensar o conceito de competência - objetivo central de formações profissionais - para além dos conhecimentos, habilidades e atitudes previamente adquiridos que prepara alguém para uma ação, mas mais como uma capacidade industriosa composta por ingredientes heterogêneos que se desenvolvem em ação e são relacionados às normas e aos valores em que as atividades industriosas humanas se desenrolam (Schwartz, 1998).

Em particular no campo da condução, veremos que as competências não se circunscrevem apenas à ação singular humana, mas, no mínimo, em uma relação entre sua ação e as reações, quase nunca controláveis, do meio em que age o condutor. Essas ações são sempre atreladas às intenções e pré-ocupações complexas que a atravessam, sejam elas em relação ao destinatário da ação, à relação ao próprio veículo e às outras atividades e necessidades da pessoa. Sendo assim, ao se circunscrever os acidentes e resultados indesejáveis do trânsito - tais como o gasto excessivo de combustíveis, infrações, transgressões e desgaste do veículo - ao comportamento do condutor, reduzem-se as possibilidades de análises e de intervenção sobre a atividade de condução profissional frente aos fenômenos do trânsito. Se mantivermos o uso dos simuladores a partir dessa lógica geral, corremos o risco de limitar de modo semelhante seu uso e eficácia. Talvez por isso se verifique em alguns textos discursos dos usuários dos simuladores queixando-se da falta de realismo das situações simuladas, não tanto em função das percepções sensório-motoras-visuais eliciadas pelo instrumento, mas pela própria condição em si do exercício realizado (Kihl et al., 2006).
Isso porque, a partir do referencial teórico da Ergonomia da Atividade e da Ergologia, os conhecimentos mais estratégicos seriam aqueles mais relacionados às situações reais que se vive no trabalho, que raramente são mobilizados nos treinamentos com esse instrumento. Portanto, para sugerir outras possibilidades do uso dos simuladores, deve-se compreender, a partir de uma análise ergológica, quais são e como se desenvolvem as competências em trânsito. A partir daí pode-se demonstrar os limites do uso desse instrumento tal qual ocorre atualmente, bem como suas possíveis possibilidades.

É importante, portanto, avançar na reflexão crítica sobre como, nos últimos anos, os simuladores de condução, sobretudo nos processos de formação profissional, vêm sendo empreendidos nas pesquisas científicas. Sugerimos, igualmente, como o ponto de vista da atividade pode apontar para outras perspectivas de investigação e implementação sobre treinamentos, mediados por simuladores. Não é nossa intenção, nesse momento, afirmar os métodos para que isso se dê. O que pretendemos, neste artigo, é apenas apontar para outros caminhos possíveis que viabilizem reflexões coletivas sobre o uso de simuladores nas práticas de formação dos profissionais dos transportes terrestres. Mas pretendemos, também, alertar para dimensões coletivas que englobam o trabalho neste setor, dimensões essas ainda pouco valorizadas nos treinamentos calcados em práticas preventivistas que superdimensionam a importância do indivíduo em um terreno de relações humanas, sociais, culturais e políticas que ultrapassam as determinações imediatas do tráfego onde esses profissionais se encontram.

\section{Algumas características das atividades de trabalho nos transportes terrestres}

A partir de pesquisa baseadas em análise ergonômica da atividade (Forzy, 2007; Hubault, 2011b; Van Elslande, 2000), podemos descrever a atividade de dirigir-conduzir veículos de transporte terrestre rodoviário como uma atividade focada em um objetivo de deslocamento, mediado por um instrumento (veículo) que possui algumas características importantes: trata-se de uma atividade multitarefas, na medida em que se realizam, ao mesmo tempo, várias tarefas relativamente distintas, compostas por tarefas principal, secundárias e anexas. Cada uma dessas tarefas impõe constrangimentos temporais peculiares que deman- 
dam solicitações visuais específicas do funcionamento perceptivo e da atenção dos condutores. Tais características de busca visual e demandas de atenção requererão deles múltiplos processos cognitivos e contínua necessidade de se realizar uma gestão dos riscos, sobretudo diante de uma diversidade de situações encontradas durante o processo de condução. Ressalta-se, além disso, que essa atividade é baseada em pouca estruturação prévia (tarefa), além de ser realizada em um meio partilhado por uma enormidade de usuários distintos, com objetivos diferentes, e que interagem entre si.

Trata-se, portanto, de uma atividade complexa, mediada por um instrumento que é reconhecidamente letal para si e para outros (Valot, 2007). Esse e outros autores (Araújo, Malloy-Diniz, \& Rocha, 2009; Hennessy, 2011) chamam a atenção, além disso, à dimensão afetiva nessa atividade, que convoca diferentes domínios dos saberes para compreender os conflitos e debates de valores que a subjazem. Entre esses valores, ilustra-se o contínuo dilema entre se respeitar a segurança - respeito às normas, proteção dos usuários dos veículos, segurança das vias - ou, paradoxalmente, valorizar a eficiência no deslocamento de corpos e objeto, impostas pelas exigências temporais e de urgência de um mundo que se dize se requer mais veloz. Enraizado nesse mundo de valores em si contraditórios, florescem dimensões de afetos e sensações também paradoxais mobilizadas pela velocidade e/ou pelo deslocamento: a ansiedade para se chegar logo em um trânsito atravancado, convive pari passo com os prazeres da velocidade, da autonomia, do encurtamento das distâncias ou da sensação de tranquilidade ao se chegar bem em seu destino almejado.

Deve-se ser cuidadoso para não se tomar cada um desses distintos aspectos citados anteriormente de modo isolado, uma vez que eles se manifestam concomitantemente, interagindo entre si a partir da gestão que cada trabalhador realiza na atividade, por meio de seus próprios recursos e usos de si (Schwartz, 2004). Do contrário, há riscos para a qualidade da transformação da atividade que se procura conhecer-transformar. Ilustrando esse aspecto, vimos anteriormente que as tarefas e conhecimentos relacionados à condução (técnico, tático e estratégico) eram manipulados nos simuladores mais ou menos independentemente uns dos outros, sendo um exemplo de como se descaracteriza a atividade real em um modelo que não encontra referência real para os usuários dos simuladores. Daí, em parte, a validação apenas parcial de transferência de conhecimento de aprendizagens singulares que, sem integração com outros conhecimentos e tarefas, correm o risco de serem ignoradas em situações reais, sempre mais complexas.

Há ainda, no caso de trabalhadores em atividade de condução, a inclusão de outras tarefas, demandas perceptivas, constrangimentos temporais, gestão dos riscos, tratamento de informações e situações que intensificam ainda mais a complexidade do que se trata essa natureza de atividade profissional. Entre elas: a extrema diversidade de situações em que o transporte, e as tarefas a ele correlatas, se dá; a partilha do meio (milieux) de trabalho com usuários não profissionais, considerando-se as profundas diferenças de competências, intenções e exigências de cada condutor nesse mesmo meio "profissional"/"não-profissional" (Valot, 2007); o prazer pela busca de sensações como a velocidade (Ledesma, Poó, \& Peltzer, 2007); as contínuas viagens que obrigam ao frequente afastamento de casa (Valot, 2007); o replanejamento permanente da rota (Moraes, 2008); as relações com os usuários e a obediência a horários prescritos em transportes regulares de passageiros (Pinto, \& Neves, 2009); fatores financeiros, organizacionais, fisiológicos, legislações, expectativa dos usuários, qualidade da via e expectativas profissionais que impactam e causam constrangimentos (contraintes) aos trabalhadores (Brunoro, Sznelwar, Bolis, \& Abrahão, 2012).

Finalmente, devem-se destacar como características da condução profissional as relações entre a atividade de trabalho nesse setor, as dimensões afetivas e psíquicas produzidas no e para o exercício da atividade e alguns de seus efeitos, seja na constituição identitária e do ofício dos trabalhadores (Moraes, Rohr, \& Athayde, 2015), seja na relação com acidentes (Lopes et al., 2012), seja na vivência dos processos saúde-doença, intensificados pelo envelhecimento dos trabalhadores (Almeida, 2002; Lancman, Sznelwar, \& Jardim, 2006).

Comparando-se as características da atividade acima, baseadas em análises ergonômicas e ergológicas, com as apresentadas em análises da tarefa realizadas por autores para se viabilizar formações por meio do uso de simuladores (Mitsopoulos-Rubens et al., 2013), verificam-se diferenças importantes na maneira de se conceber a atividade, bem como o papel da gestão do trabalho por parte dos profissionais. Se, por um lado, nos modelos tradicionais de análise das tarefas 
(Brannick, Levine, \& Morgeson, 2007) evidencia-se um papel menos ativo dos trabalhadores no processo de gestão cotidiana da atividade, por outro, a partir das análises da atividade real, verifica-se que a gestão do trabalho, em sua dimensão microgestionária, não é um privilégio em domínio da gerência, mas um aspecto inevitável e fundamental da atividade humana (Schwartz, 2004).

Nessa direção, podemos ilustrar a partir das observações acima, alguns processos de gestão das variabilidades cotidianas, sempre complexas, ligados à atividade do condutor e que requerem contínuos usos-de-si no processo da condução.

Antecipação contínua dos deslocamentos em trânsito: é sempre impreciso antecipar com segurança o que se fará nas estradas. A atenção e cálculos mentais, mesmo quando não totalmente conscientes, são ininterruptamente requeridos, acarretando em fadiga física e mental. Para se realizar de modo adequado essa contínua antecipação, em meio a uma extrema variabilidade de situações, potencializadas pelas exigências do trabalho sob temporalidades precisas, sobretudo em transportes coletivos e de mercadorias, em meio a outros usuários das vias, demanda-se do condutor competências sempre mais ou menos situadas, ou seja, conhecimentos adquiridos cotidianamente nas estradas, possibilitando aos trabalhadores um agir competente (Schwartz, 2009). O objetivo de reduzir a variabilidade, simplificar o trabalho, conforme preconizava a ficção taylorista, seria, aqui, uma solução impossível (Schwartz, 2004).

Formar um expert nesse complexo contexto profissional não pode ser pensado sem essa relação antecipação-variabilidade em ato que abrange todos os níveis e tarefas envolvidas na condução. Assim, uma formação cujo foco é desenvolver uma habilidade tomada isoladamente será sempre parcialmente insuficiente, uma vez que em situações reais de trabalho essa habilidade se relacionará, inevitavelmente, a uma rede complexa de outras habilidades e conhecimentos, influenciando-se reciprocamente. Ademais, deve-se reconhecer que a competência de condução não é apenas aquisição de capacidades cognitivas individuais, mas fruto da produção e desenvolvimento de um coletivo que contribui para produção e partilha de sentido e que fornece instrumentos para superar essa defasagem antecipação-variabilidade em atividade (Hubault, 2011a; Schwartz, 2009). Daí o limite do uso de simuladores em forma- ções individuais que se restringem ao adestramento do corpo e da mente do condutor para desenvolver habilidades isoladas em cenários simulados que desconsideram deliberadamente outras condições da atividade que, em situações reais de trabalho, não podem jamais serem desconsiderados pelo condutor e que, por isso, influenciam em seu comportamento bem como contribuem para as inúmeras variabilidades cotidianas. Para ilustrar, basta observarmos o efeito que um atraso temporal produz nos modos de condução de boa parte dos motoristas profissionais.

Usos singulares do corpo: em alguns veículos, o acoplamento corpo-máquina é de tal ordem que é difícil não pensar no prolongamento vivo, cerebral, do corpo nas máquinas (no caso das motocicletas, isso é muito mais manifesto). $\mathrm{O}$ uso do termo pilotagem por parte de alguns profissionais parece emblemático para ilustrar esse acoplamento (Moraes, 2008). Os modos como um condutor se mobiliza de corpo inteiro nesses processos são claramente evidentes em situação real. Porém, estudos com simuladores frequentemente carecem da capacidade de apreender toda a complexidade corporal envolvida no processo de condução (Carsten, \& Jamson, 2011). Até porque não é apenas o corpo biológico que se mobiliza nesta atividade: em situações reais, o corpo é mobilizado em conjunto com sua história, seus conhecimentos, saberes e relações, bem como suas dimensões psíquicas e afetivas. Compreender os usos que se fazem do corpo-si (Schwartz, 2011) parece aqui fundamental para ampliar o escopo das mobilizações nos simuladores (Moraes, \& Pinto, 2011), a despeito da insuficiência técnica que limita a aplicabilidade desses instrumentos (Fisher et al., 2011).

A condução dos veículos em situações de trabalho: a despeito de ser realizada na maioria das vezes solitariamente, esta é uma atividade inevitavelmente coletiva, requerendo a mobilização de saberes relacionais e a gestão das redes de solidariedade (suporte, como pares e pontos de apoio), de controle (gerência, expectativas dos clientes) e de partilha (usuários das vias). Aspecto pouco discutido em pesquisas com tais trabalhadores, os atravessamentos coletivos na atividade de trabalho são fundamentais tanto na contribuição da execução das tarefas quanto na produção de processos identitários (Velôso, Oliveira Filho, Medeiros, \& Araújo, 2009). Assim, é importante que as características da tarefa e do processo de condução, acima elencadas, sejam apreendidas também a partir do com- 
plexo de sentidos, valores e saberes, sempre sociais, que coletivamente os trabalhadores dão a elas. Aqui, o conceito schwartzniano de entidade coletiva relativamente pertinente (ECRP) (Schwartz, \& Durrive, 2010) permite compreender essas relações, sempre provisórias, mas em busca de pertinência, de modo a não se limitar apenas nas relações imediatas entre pares. Esta é uma expressão utilizada em Ergologia para expressar que trabalhar é sempre também viver junto com outrem, de tal maneira que ao trabalhar há um fazer, refazer e desfazer contínuo existente em toda organização humana, inscrito em sua própria história num (re) trabalho "dos valores que nos anima a todos, na nossa vida social" (Schwartz, \& Durrive, 2010, p. 154). Abordar a atividade de condução como se fosse individual é não acessá-la em sua integralidade.

Verificamos, portanto, uma complexa rede de fatores que se deve levar em conta para se compreender $\rightarrow$ transformar a atividade de trabalho nos transportes terrestres. Reiteramos que a atividade de trabalho nesse setor é sempre intensamente uma atividade de gestão (Schwartz, 2004), e que é crucial o papel dos coletivos em meio à atividade. A extensão dos efeitos dessas dimensões coletivas nos transportes rodoviários está ainda a se compreender, embora algumas pesquisas já tenham demonstrado alguns aspectos de sua importância na profissão dos motoboys (Diniz et al., 2005; Moraes, 2008). Tais pesquisas demonstraram, porém, que, apesar de importante, esses suportes coletivos eram, nos casos analisados, ainda frágeis, gerando limitações da abrangência dos efeitos das ECRP na atividade de trabalho, o que requereria seu fortalecimento, por meios difíceis de serem completamente previsíveis. Esse processo de formação do coletivo deve se dar, de qualquer modo, desde o início das atividades de um trabalhador na profissão, uma vez que ele é inserido em uma atividade perigosa desde os primeiros dias de trabalho.

Além da dimensão coletiva, a dimensão afetiva também tem um papel amplificado nas atividades de trabalho nesse setor. Essa dimensão é mobilizada continuamente na medida em que a condução é exercida em meio a diferentes exigências, que acarretam em mobilização afetiva dos trabalhadores para enfrentar as incompatibilidades dessas exigências e as condições que o meio fornece, tais, como, por exemplo, a necessidade de realizar entregas em um trânsito engarrafado. Além desse aspecto, a partir de contribuições de outras clínicas do trabalho, articuladas aqui a partir da Ergologia, podemos afirmar que a dimensão afetiva dos trabalhadores exerce uma função psicológica no processo de produção de si a partir do trabalho, na qual o sujeito se desenvolve reconhecendo-se naquilo que faz (Clot, 2010). Neste sentido, entendemos que há um campo insuficientemente explorado e ocupado por psicólogos do trabalho, que poderiam oferecer sua competência para favorecer as análises do trabalho que visam o desenvolvimento de estilos e gêneros profissionais dos trabalhadores em questão. Pesquisas com trabalhadores dos transportes rodoviários sugerem uma afinidade entre condutores profissionais e seu trabalho, de modo que se verifica uma extrema positividade dessas profissões no que tange à mobilização afetiva e sua contribuição nos processos identitários (Moraes et al., 2015; Pinto, \& Neves, 2009). Nenhum dos estudos citados no item anterior deste artigo abordam essas dimensões, que avaliamos fundamentais no processo de condução e que, aliados às dimensões coletivas e aos valores subjacentes à atividade, são centrais para se compreender porque a execução desse trabalho é ou não eficiente e ao mesmo tempo seguro.

\section{Competências a serem desenvolvidas pelo público alvo, métodos empregados e atuação dos formadores}

Diante da enigmática rede de elementos e dimensões que são mobilizadas na atividade profissional de transporte rodoviário, as ferramentas de transformação dessas atividades são sempre muito complexas, tornando sempre ambíguo o papel da formação profissional. Na medida em que é possível apropriar-se de diversos modos dos riscos enfrentados pelos trabalhadores em suas atividades (Hubault, 2011a), o papel da formação terá enquadramentos distintos. De um lado, verifica-se a pertinência e necessidade de se pensar o problema dos acidentes e riscos nos transportes rodoviários terrestres não apenas em termos de exposição a riscos prévios e cognoscíveis - como a velocidade, jornada de trabalho, nível de atenção, desenho das pistas - mas também em termos de um engajamento singular entre um trabalhador, suas exigências e dimensões concretas quase incompossíveis, em cenários sempre variáveis (Van Elslande, 2000). A primeira postura conduz à necessidade pedagógica de se qualificar os trabalhadores para estar atento aos riscos e saber se comportar adequadamente 
diante deles. A partir daí, sua qualificação profissional para desenvolvimento de competências diretamente relacionadas ao trânsito, tais como destreza veicular, concepção e uso de estratégias de busca e escaneamento visual e identificação e antecipação de situações de risco são as estratégias mais pertinentes. Considera-se, nessa perspectiva, a possibilidade, ou mesmo a exigibilidade, de comportamentos que se adaptem às normas previamente estabelecidas, sejam elas calcadas nas leis de trânsito, em princípios econômicos ou em valores instrucionais, como os da direção defensiva. Para atender a esses pressupostos, geralmente se propõe como estratégia para redução dos acidentes, as formações individuais baseadas em saberes e práticas definidas a priori. Alia-se a esses processos de formação, a inclusão, no sistema homem-máquina, de instrumentos capazes de controlar os comportamentos, considerados de risco, em busca da proteção geral dos próprios trabalhadores, tais como o uso de tacógrafos, monitoramento por satélites ou vídeo e o desenvolvimento de dispositivos de controle da atenção/distração (Victor et al., 2011). Neste modelo, no fim das contas, é a condução, e não o trabalho, que importa gerenciar: a atividade de condução não sendo necessariamente apreendida como atividade de trabalho (Hubault, 2011a), generalizando esses princípios para quaisquer situações de condução, independentemente da situação profissional. $\mathrm{O}$ uso dos simuladores seria, a partir dessa postura, um instrumento que permitiria treinar os trabalhadores para enquadrarem-se nesse ideal previamente identificado em tarefas analisadas com instrumentos científicos, o que acarretaria em efeitos ideais e produtivos. Como tentamos mostrar, apontando inclusive alguns limites, parece-nos que a literatura atual dos simuladores de condução para os transportes terrestres defende uma postura que se encontra nesse polo de possibilidades.

Em contrapartida, outra postura teórico-epistemológica acerca da atividade de condução profissional, que entende o risco como efeito de um engajamento homem-máquina-ambiente, remete ao modo como cada condutor, em seus dilemas cotidianos e intensas variabilidades, faz face às distintas normas e demandas para dar conta dessas questões. Aqui, são menos os saberes em si que falham, mas a impossibilidade de mobilizá-los diante cenários específicos e sempre parcialmente singulares (Van Elslande, 2000). A produção desses cenários envolve, além dos saberes profissionais, as políticas organizacionais, os modos de recrutamento, remuneração e gerenciamento dos trabalhadores, as políticas de gestão das malhas viárias, as diversas e conflituosas culturas subjacentes aos mais diversos usuários das vias, incluindo a dos condutores trabalhadores, os destinatários das cargas e pessoas transportadas, entre outros. Diante desse contexto, a formação deixa de ser focada em habilidades e conhecimentos isolados e recai igualmente sobre a necessidade de efetuar-se por e para análises do trabalho (Teiger, \& Lacomblez, 2013), requerendo um debruçar clínico sobre cada cenário, donde se extrai o conjunto de exigências e (in)compatibilidades mobilizadas pelos trabalhadores em ação.

Na medida em que a atividade de condução como atividade de trabalho não se resume à utilização de um veículo (Hubault, 2011a), sugere-se, portanto, o desenvolvimento de processos de formação-prevenção por meio de dispositivos de pares que possibilitam a partilha de experiências que permitam desenvolver a capacidade de ação de cada um por meio da ampliação dos saberes coletivos (Hubault, 2011a). Se atualmente não se usa os simuladores de condução com esse propósito, não significa que não se possa fazê-lo: uma longa tradição francesa de usos de simulação para o treinamento, sobretudo ligados à Didática Profissional (Pastré, 2011), vem realizando formação de profissionais das áreas de saúde, de pilotagem de usinas nucleares e siderúrgicas, de trabalhadores rurais e de pilotos de avião, demonstrando possibilidades do uso de simuladores como instrumentos de análise do trabalho e de formação em situação laboral (Amalberti, 2013; Fauquet-Alekhine, \& Pehuet, 2011; Pastré, 2005a).

No que tange ao modo de se apropriar da atividade de condução e os riscos a ela atrelados a partir do conceito de exposição aos riscos, verificam-se evidências de que os simuladores contribuem, ao menos parcialmente, para a aquisição de algumas competências importantes: aprendizagem inicial da condução, economia de combustível e desenvolvimento de competências de percepção e antecipação de situações de risco (Moraes, 2016). Ressalta-se, entretanto, que estas são formações que não se debruçam sobre as singularidades em si, mas sobre as generalizações da condução que não fornecem todas as ferramentas necessárias para que os trabalhadores, em atividade, consigam realizar suas tarefas (e não somente, conduzir um veículo). Nesse sentido, o uso de simuladores em processos de formação que reconheçam 
os acidentes enquanto problemas do (meio de) trabalho nos engajamentos homem-ambiente, mediado pela máquina, ainda estão a ser inventados. O que se destaca desse ponto de vista é justamente reconhecer a realidade do trabalho no qual se debruçam esses trabalhadores, mais que simplesmente um processo generalizável condutor-veículo-tráfego-vias.

Com base nas referências do patrimônio teórico-epistemológico da Ergonomia da Atividade, que parte da análise do trabalho para produzir transformações positivas em um meio, de modo a conciliar qualidade, produtividade, saúde e segurança, e da Ergologia, que desenvolve seus saberes e suas técnicas de intervenção exatamente a partir do reconhecimento dos inevitáveis usos do corpo-si (Schwartz, 2011) entre o que se prevê e o que se executa, podemos encontrar um terreno fértil que permite a articulação de ambos os aspectos acima considerados, mais ou menos como encontramos no acumulado da experiência da simulação em saúde (Amalberti, 2013). Experiências ainda mais antigas, baseadas em modos mais rudimentares do que hoje poderíamos chamar de simulação, como a técnica do sósia (Oddone, Re, \& Briante, 1981; Schwartz, 2000), confirmam as viabilidades possíveis para o processo de simulação como meios de análise por e para formação do trabalho. Se, de um lado, tais referências teóricas orientam caminhos para se reconhecer as defasagens entre o que se antecipa e o que se realiza, por outro lado, elas conduzem ao reconhecimento inevitável de que não é senão por meio dos saberes-fazeres dos trabalhadores que se têm as ferramentas para se transformar a realidade (Falzon, 2007).

A partir desse referencial teórico pode-se constatar que a simulação é sempre uma antecipação parcial da atividade-fim. Ao mesmo tempo, deve-se reconhecer que, como qualquer atividade, viver a experiência da simulação é também uma atividade em si, na qual se incluem todas as defasagens acima apontadas. Na medida em que se vive uma atividade de simulação, que não é nunca a atividade real do trabalho, mas nem por isso menos importante para o desenvolvimento profissional, deve-se evidenciar ali a complexidade de elementos que mobilizam o corpo dos condutores para a ação, considerando-se que se trata de um corpo biológico, psicológico, social e histórico, em uma palavra, um corpo-si (Schwartz, 2011). Ao se reconhecer que a atividade de simulação é uma atividade que mobiliza um corpo-si, devemos estar atentos a todos os aspectos que o mobilizam na experiência simulada. Daí, então, que o desenho de formações profissionais por meio de simuladores considerando-se aspectos usualmente não reconhecidos nas pesquisas internacionais, entre os quais as dimensões afetivas e psíquicas, talvez venha a propiciar a adequação dos simuladores para a realidade profissional no Brasil. Assim, sugere-se que estes treinamentos se deem sobre análises da atividade, mobilizando-se o corpo-si em ato, em todas as suas dimensões: biológica, psicológica, social e histórica, reconhecendo-se o eixo coletivo de sua produção. Aqui, abre-se um importante campo de trabalho na qual a prática e os saberes da psicologia são imprescindíveis. Alguns princípios sobre tais possibilidades de formação, a partir da perspectiva ergológica, foram propostos (Durrive, \& Jacques, 2010), embora não se encontre neste texto referências ao uso de simuladores como instrumentos de suporte, o que nos abre um campo de pesquisas e experimentações futuras.

\section{Considerações finais}

Consideramos que uma das vias possíveis para que os processos de formação por meio de simuladores possam ser mais efetivos seria a realização de formações que abordem e problematizem as dimensões afetivas e psíquicas envolvidas em situações de condução profissional. O uso de cenários simulados que envolvam algum tipo de jogo, por exemplo, pode ser alternativa que mobilize os trabalhadores a experimentar, ainda que simuladamente, as sensações e mobilizações afetivas e psíquicas que se vivencia em situações de trabalho real. O uso de jogos em formações profissionais com simuladores já foi realizado com relativo sucesso, mesmo que tais experimentações tenham sido realizadas a partir de outra perspectiva teórica das mencionadas no presente artigo (Bonch-Bruevich, Kremez, \& Chirkov, 2010). De todo modo, os saberes da Psicologia são aqui fundamentais.

Outra via possível para que formações profissionais com simuladores tenham efeitos mais duradouros e efetivos no trabalho dos profissionais do setor seria que tais formações envolvessem a análise coletiva da atividade, destacando-se o uso da prática do que Pastré denomina de debriefing, que são as "sessões coletivas de análise da atividade sob a direção de instrutores", realizadas em situações de formação profissional com uso de simuladores ou outros dispositivos de aná- 
lise da situação (Pastré, 2005b). A partir da relevância da presença das dimensões coletivas e relacionais da gestão de uma instalação, como em toda indústria de processo, este debriefing mobiliza a dimensão coletiva largamente ignorada nos usos das formações profissionais com simuladores, seja evidenciando a tomada de consciência dos problemas e consequência das ações, estratégia de debriefingmais utilizada entre os aprendizes; seja para pôr em relevo e análise as estratégias utilizadas para executar uma ação, estratégia de debriefing mais utilizada entre os experts.

A análise coletiva do trabalho em situações de simulação é, portanto, uma via que tem pertinência e possibilidades, sobretudo se considerarmos os instrutores menos como experts capazes de transmitir conhecimentos, como se transfere um software numa conexão digital, e mais como portadores de conceitos quem contribuem para uma mobilização potente de reflexões sobre o trabalho, a situação e a atividade. Assim, articulando-se instrutores e trabalhadores, ambos amparados por uma postura ética de respeito aos saberes e experiências de quem vive cotidianamente o trabalho, forma-se um dispositivo de análise da atividade denominado de dispositivo dinâmico de três polos (Schwartz, 2000), que visa à compreensão e à transformação das situações do trabalho por, para e com os trabalhadores. Esse dispositivo destaca-se como um possível suporte ético-epistemológico na base dessa análise coletiva das situações simuladas em formação profissional.

Porém, mais que isso: essa formação contribuiria para o desenvolvimento do próprio ofício. Para viabilizar esse processo, as análises da atividade mediadas por pessoas experientes que valorizam a produção desse coletivo nos parece ser uma proposta promissora, pelo fato de que não se trata apenas de realizar treinamentos sobre tarefas e normas de segurança, mas de colocar em debate, o mais próximo possível da atividade, as competências, ambiguidades e dilemas enfrentados pelos trabalhadores, sobretudo a partir dos conhecimentos, valores e suportes produzidos coletivamente sobre a atividade (Durrive, \& Jacques, 2010).

Nessa linha argumentativa, o uso de simuladores como instrumento mobilizador poderia contribuir como importante estratégia de formação, sobretudo de novos trabalhadores, porque propiciaria um processo de análise coletiva da atividade visando o desenvolvimento profissional e do próprio ofício, sem o enfrentamento cotidiano dos riscos profissionais, mas indo além das clássicas formações realizadas sobre a tarefa. Pesquisas futuras nessa direção podem verificar a pertinência dessas hipóteses. Talvez a partir daí novas ferramentas sejam produzidas, contribuindo para orientação do uso de simuladores durante a formação desses profissionais.

\section{Referências}

Allen, R. W., Park, G. D., Cook, M. L., \& Fiorentino, D. (2007). The effect of driving simulator fidelity on training effectiveness. In Driving Simulation Conference, North America 2007 (DSC-NA 2007), Iowa City IA.

Almeida, N. D. V. (2002). Contemporaneidade x trânsito reflexão psicossocial do trabalho dos motoristas de coletivo urbano. Psicologia: Ciência e Profissão, 22(3), 62-69. https://doi.org/10.1590/S1414-98932002000300010

Amalberti, R. (2013). Gestion des risques et facteurs humains: rôle de la simulation. In S. Boet, J.-C. Granry, \& G. Savoldelli (Eds.), La simulation en santé: de la théorie à la pratique (pp. 3-14). Paris: Springer.

Araújo, M. M., Malloy-Diniz, L. F., \& Rocha, F. L. (2009). Impulsividade e acidentes de trânsito. Archives of Clinical Psychiatry (São Paulo), 36(2), 60-68. https://doi.org/10.1590/S0101-60832009000200004

Bacchieri, G., \& Barros, A. J. D. (2011). Acidentes de trânsito no Brasil de 1998 a 2010: muitas mudanças e poucos resultados. Revista de Saúde Pública, 45(5), 949-963. https://doi.org/10.1590/S0034-89102011005000069

Blanco, M., Hickman, J. S., Hanowski, R. J., \& Morgan, J. F. (2011). The commercial driver. In D. L. Fisher, M. Rizzo, J. K. Caird, \& J. D. Lee (Eds.), Handbook of driving simulation for engineering, medicine and psychology (pp. 31(1-12)). Boca Raton: CRC Press.

Boet, S., Jaffrelot, M., Naik, V. N., Brien, S., \& Granry, J. C. (2014). La simulation en santé en Amérique du Nord: état actuel et évolution après deux décennies. Annales Françaises d'Anesthésie et de Réanimation, 33(5), 353-357. https:// doi.org/10.1016/j.annfar.2014.03.004 
Bonch-Bruevich,V.V.,Kremez,A.S.,\&Chirkov,B.P.(2010).Profession-relevantskillsdevelopmentusingpsychophysiological trainer. Automation and Remote Control, 71(7), 1461-1464. https://doi.org/10.1134/S0005117910070222

Brannick, M., Levine, E. L., \& Morgeson, F. P. (2007). Job and work analysis methods, research, and applications for human resource management. Thousand Oaks. CA: Sage.

Brasil. (2014). Ministério das Cidades. Departamento Nacional de Trânsito. Resolução no 493, de 5 de junho de 2014. Altera a Resolução CONTRAN no 168, de 14 de dezembro de 2004, que trata das normas e procedimentos para a formação de condutores de veículos automotores e elétricos e a Resolução CONTRAN no 358 , de 13 de agosto de 2010, que trata do credenciamento de instituições ou entidades públicas ou privadas para o processo de formação de condutores. Diário Oficial da União, 6 de junho de 2014.

Brasil. (2015). Ministério das Cidades. Departamento Nacional de Trânsito. Resolução no 543, de 15 de julho de 2015. Altera a Resolução CONTRAN no 168 , de 14 de dezembro de 2004, com a redação dada pela Resolução CONTRAN no 493, de 05 de junho de 2014, que trata das normas e procedimentos para a formação de condutores de veículos automotores e elétricos.Diário Oficial da União, 20 de julho de 2015.

Brock, J. F., Jacobs, C., \& Buchter, R. (2001). Design of a guidebook for the acquisition and use of driving simulators for training transit bus operators. In Proceedings of the First International Driving Symposium on Human Factors in Driver Assessment, Training and Vehicle Design (pp. 339-344), Aspen, CO.

Brook-Carter, N., Luke, T., \& Parkes, A. (2004). Evaluating the use of a truck simulator for training drivers in safe and fuel efficient driving. In Proceedings of the European Transport Conference (ETC) (p. 13), Strasbourg, France.

Brunoro, C., Sznelwar, L. I., Bolis, I., \& Abrahão, J. (2012). Contributions of ergonomics to the construction of bus drivers health and excellence in public transport and at work. Work, 41(Suppl 1), 30-35. https://doi.org/10.3233/WOR-2012-0131-30

Carsten, O., \& Jamson, A. H. (2011). Driving simulators as research tools in traffic psychology. In B. E. Porter (Ed.), Handbook of traffic psychology (pp. 87-96). San Diego: Elsevier.

Clot, Y. (2010). Poder de agir. Belo Horizonte: FabreFactum.

Diniz, E. P. H., Assunção, A. Á., \& Lima, F. P. A. (2005). Prevenção de acidentes: o reconhecimento das estratégias operatórias dos motociclistas profissionais como base para a negociação de acordo coletivo. Ciência \& Saúde Coletiva, 10(4), 905-916. https://doi.org/10.1590/S1413-81232005000400014

Dorn, L., \& Stannard, J. (2006). Simulator performance differences between experienced and novice bus drivers. Advances in Transportation Studies, (Special Issue), 45-52.

Durrive, L., \& Jacques, A.-M. (2010). O formador ergólogo ou "ergoformador": uma introdução à ergoformação. In Y. Schwartz \& L. Durrive (Eds.), Trabalho \& ergologia: conversas sobre a atividade humana (2a ed., pp. 295-318). Niterói, RJ: EDUFF.

European Parliament. (2003) Directive 2003/59/EC, 15 July 2003. Initial qualification and periodic training of drivers of certain road vehicles for the carriage of goods or passengers, amending Council Regulation (EEC) No 3820/85 and Council Directive 91/439/EEC and repealing Council Directive 76/914/EEC. Official Journal of the European Union, L 226/4.

Falzon, P. (Ed.). (2007). Ergonomia. São Paulo, SP: Edgar Blucher

Fauquet-Alekhine, P., \& Pehuet, N. (Eds.). (2011). Améliorer la pratique professionnelle par la simulation. Toulouse: Octarès.

Fisher, D. L., Rizzo, M., Caird, J. K., \& Lee, J. D. (Eds.). (2011). Handbook of driving simulation for engineering, medicine, and psychology. Boca Raton, FL: CRC Press.

Forzy, J.-F. (2007). Condução de automóveis e concepção ergonômica. In P. Falzon (Ed.), Ergonomia (pp. 557-571). São Paulo, SP: Blucher.

Foucault, M. (2009). Vigiar e punir: nascimento da prisão. Petrópolis: Vozes.

Goode, N., Salmon, P. M., \& Lenné, M. G. (2013). Simulation-based driver and vehicle crew training: Applications, efficacy and future directions. Applied Ergonomics, 44(3), 435-444. https:// doi.org/10.1016/j.apergo.2012.10.007

Grüneberg, U., \& Schröder, A. (2012). Status of simulator training for professional drivers in Europe. Project SIMTEB: Simulator-based training for European bus drivers. Dortmund: European Comunity. 
Hennessy, D. (2011). Social, personality, and affective constructs in driving. In B. E. Porter (Ed.), Handbook of traffic psychology (pp. 149-163). London: Elsevier.

Hubault, F. (2011a). Les enjeux scientifique, organisationnels et politiques. In F. Hubault (Ed.), Activité de conduite, activité de travail. Toulouse: Octares.

Hubault, F. (Ed.). (2011b). Activité de conduite, activité de travail. Toulouse: Octares.

Kihl, M., Herring, D., Wolf, P., McVey, S., \& Kovuru, V. (2006). Snowplow simulator training evaluation. Phoenix: Arizona Department of Transportation.

Lancman, S., Sznelwar, L. I., \& Jardim, T. A. (2006). Sofrimento psíquico e envelhecimento no trabalho: um estudo com agentes de trânsito. Revista de Terapia Ocupacional da Universidade de São Paulo, 17(3), 129-136. https://doi.org/10.11606/issn.2238-6149.v17i3p129-136

Ledesma, R., Poó, F., \& Peltzer, R. (2007). Búsqueda impulsiva de sensaciones y comportamiento de riesgo en la conducción. Avaliação Psicológica, 6(2), 117-125. Recuperado de http://pepsic.bvsalud.org/scielo.php?scrip$\mathrm{t}=$ sci_arttext\&pid=S1677-04712007000200002

Lopes, M. G. R., Vilela, R. A. G., Almeida, I. M., Mioto, O. L., Takahashi, M. A. B. C., \& Perin, F. O. (2012). Tragedy on grade crossing: driver failure or systemic fragility? Work, 41(Suppl 1), 3148-3154. https://doi.org/10.3233/WOR-2012-0576-3148

Lucas, F. R., Russo, L. E. A., Kawashima, R. S., Figueira, A. C., Larocca, A. P. C., \& Kabbach Junior, F. I. (2013). Uso de simuladores de direção aplicado ao projeto de segurança viária. Boletim de Ciências Geodésicas, 19(2), 341-352. https:// doi.org/10.1590/S1982-21702013000200010

Masciocchi, C., Dark, V., \& Parkhurst, D. (2006). Evaluation of virtual reality snowplow simulator training: a literature review. Ames Iowa: Department of Transportation.

Mendes, L. G., Mendes, E. A. G., \& Bomfin, D. (2013). Simuladores de condução de trens: processo de aprendizagem de maquinistas ferroviários. In $6^{\circ}$ Congresso Nacional de Ambientes Hipermígia para Aprendizagem: Tecnologias Contemporâneas, Acessibilidade Digital e seus Desafios, João Pessoa, PB.

Mitsopoulos-Rubens, E., Lenné, M. G., \& Salmon, P. M. (2013). Driver simulation-based training of heavy vehicle operators: Targeted task analysis and considerations for training design. In Proceedings of the 7th International Driving Symposium on Human Factors in Driver Assessment, Training, and Vehicle Design (pp. 418-424). New York, NY.

Moraes, T. D. (2008). Coletivo de trabalho e atividade dos motoboys: gênero profissional, saberes operatórios e riscos da atividade de trabalho (Tese de doutorado). Universidade do Estado do Rio de Janeiro, Rio de Janeiro.

Moraes, T. D. (2016). O uso de simuladores em atividades de formação para profissionais dos transportes rodoviários. Ciencia \& Trabajo, 18(55), 48-57. https://doi.org/10.4067/S0718-24492016000100009

Moraes, T. D., \& Pinto, F. M. (2011). O corpo nas atividades em trânsito: condutores profissionais e mobilização do corpo-si. Cadernos de Psicologia Social do Trabalho, 14(2), 279-294. https://doi.org/10.11606/issn.1981-0490.v14i2p279-294

Moraes, T. D., Rohr, R. V., \& Athayde, M. (2015). Ingresso, permanência e abandono na profissão de motoboys: constituição de si e da profissão. Laboreal, 11(1), 69-83. https://doi.org/10.15667/laborealxi0115tdm

Morgan, J. F., Tidwell, S. A., Medina, A., Blanco, M., Hickman, J. S., \& Hanowski, R. J. (2011). Commercial motor vehicle driving simulator validation study: Phase II. Washington, DC: US Departament of Transportation.

National Center for Transit Research - NCTR. (2013). A qualitative analysis of bus simulator training on transit incidents: a case study in Florida. Florida: National Center for Transit Research.

Neukum, A., Lang, B., \& Krueger, H.-P. (2003). A simulator-based training for emergency vehicle driving. In Proceedings of the Driver Simulation Conference, North America, Dearborn, Michigan.

Oddone, I., Re. A., \& Briante, G. (1981). Redécouvrir l'expérience ouvrière. Paris: Messidor.

Pastré, P. (2005b). Apprendre par la résolution de problèmes : le rôle de la simulation. In P. Pastré (Ed.), Apprendre par la simulation: de l'analyse du travail aux apprentissages professionnels (pp. 17-40). Toulouse: Octerès.

Pastré, P. (Ed.). (2005a). Apprendre par la simulation: de l'analyse du travail aux apprentissages professionnels. Toulouse: Octares.

Pastré, P. (2011). La didactique professionnelle: approche anthropologique du développement chez les adultes. Paris: PUF. 
Pinto, F. M., \& Neves, M. Y. (2009). A gestão da atividade do motorista de ônibus: um olhar ergológico. Estudos e Pesquisas em Psicologia, 9(2), 493-511. Recuperado de http://www.revispsi.uerj.br/v9n2/artigos/pdf/v9n2al5.pdf

Sampaio, J. R. (1998). Psicologia do trabalho em três faces. In I. B. Goulart (Ed.), Psicologia do trabalho e gestão de recursos humanos (pp. 19-40). São Paulo, SP: Casa do Psicólogo.

Santana, V., Nobre, L., \& Waldvogel, B. C. (2005). Acidentes de trabalho no Brasil entre 1994 e 2004: uma revisão. Ciência \& Saúde Coletiva, 10(4), 841-855. https:// doi.org/10.1590/S1413-81232005000400009

Schwartz,Y.(2000).Acomunidadecientíficaampliadaeoregimedeproduçãodesaberes. TrabalhoeEducação, 7,38-46. Recuperado de http://www.portal.fae.ufmg.br/seer/index.php/trabedu/article/view/1681/1274

Schwartz, Y. (1998). Os ingredientes da competência: um exercício necessário para uma questão insolúvel. Educação \& Sociedade, 19(65), 101-140. https://doi.org/10.1590/S0101-73301998000400004

Schwartz, Y. (2011). Pourquoi le concept de corps-soi? Corps-soi, activité, expérience. Travail et Apprentissages, (7), 148-177.

Schwartz, Y. (2009). Produzir saberes entre aderência e desaderência. Educação Unisinos, 13(3), 264-273. https://doi.org/10.4013/edu.2009.133.09

Schwartz, Y. (2004). Trabalho e gestão: níveis, critérios, instâncias. In M. Figueiredo, M. Athayde, J. C. Brito, \& D. Alvarez (Eds.), Labirintos do trabalho (pp. 23-33). Rio de Janeiro, RJ: DP\&A.

Schwartz, Y., \& Durrive, L. (Eds.). (2010). Trabalho e ergologia: conversas sobre a atividade humana. Niterói, RJ: Eduff.

Spector, P. (2002). Psicologia nas organizações. São Paulo, SP: Saraiva.

Strayer, D. L., \& Drews, F. A. (2003). Simulator training improves driver efficiency: Transfer from the simulator to the real world. In 2nd International Driving Symposium on Human Factors in Driver Assessment, Training and Vehicle Design (pp. 190-193), Utah.

Taylor, F. W. (2004). Scientific management. New York, NY: Routledge.

Teiger, C., \& Lacomblez, M. (Eds.). (2013). (Se) Former pour transformer le travail: dynamiques de constructions d'une analyse critique du travail. Laval: Presses de l'Université Laval.

Uhr, M. B. F., Felix, D., Williams, B. J., \& Krueger, H. (2003). Transfer of training in an advanced driving simulator: comparison between real world environment and simulation in a manoeuvring driving task. In DSC North America 2003 Proceedings, Dearborn, Michigan .

Valot, C. (2007). O transporte, a segurança e a ergonomia. In P. Falzon (Ed.), Ergonomia (pp. 573-586). São Paulo, SP: Edgar Blucher.

Van Box Som, A. (2010). Des sciences humaines aux sciences de l'ingénieur (comportements humains, activités finalisées et conception de systèmes d'assistance à la conduite de véhicules industriels). Lyon: Université Lumière Lyon.

Van Elslande, P. (2000). Lerreur humaine dans les scénarios d'accident cause ou conséquence? Recherche Transports - Sécurité, 66, 7-31. https://doi.org/10.1016/S0761-8980(00)90002-5

Velôso, T. M. G., Oliveira Filho, P., Medeiros, C. S., \& Araújo, A. S. (2009). A identidade em discursos de taxistas. Psicologia \& Sociedade, 21(1), 118-127. https://doi.org/10.1590/S0102-71822009000100014

Victor, T., Hickman, J., Camden, M., Jarlengrip, J., Larsson, C., Morgan, J. et al. (2011). U34: driver distraction: an inattention-mitigation component for behavior-based safety programs in commercial vehicle operations (IM-BBS). Washington, DC: National Transportation Research Center.

World Health Organization. (2013). Global status report on road safety 2013: supporting a decade of action. Geneva: World Health Organization.

\section{Thiago Drumond Moraes}

Doutor em Psicologia Social pela Universidade do Estado do Rio de Janeiro, Rio de Janeiro - RJ. Docente da Universidade Federal do Espírito Santo, Vitória - ES. Brasil.

E-mail: thiago.moraes@ufes.br 


\section{Paulo César Zambroni-de-Souza}

Doutor em Psicologia Social pela Universidade do Estado do Rio de Janeiro, Rio de Janeiro - RJ. Docente da Universidade Federal da Paraíba, João Pessoa - PB. Brasil.

E-mail: paulozamsouza@yahoo.com.br

Yves Schwartz

Professor emérito da Aix-Marseille Université, Institute d'Ergologie, Université d'Aix-Marseille, CEPERC-CNRS, UMR 7304 Aix-Marseille Université. França.

E-mail: yves.schwartz@univ-amu.fr

Endereço para envio de correspondência:

Universidade Federal do Espírito Santo, Centro de Ciências Humanas e Naturais, Departamento de Psicologia Social e do Desenvolvimento.

Av. Fernando Ferrari, 514. Campus Universitário de Goiabe CEP: 29075-910.

Vitoria - ES. Brasil.

Recebido 25/09/2015

Aprovado 14/12/2016

Received 09/25/2015

Approved 12/14/2016

Recibido 25/09/2015

Aceptado 14/12/2016

Financiadores:

CAPES (Thiago Drumond Moraes Bolsista, processo n. BEX 2103/14-1) e FAPES

Como citar: Moraes, T. D., Zambroni-de-Souza, P. C., \& Schwartz, Y. (2017). Os usos de simuladores durante a formação profissional para a condução de transportes rodoviários. Psicologia: Ciência e Profissão, 37(1): 192-207. https://doi.org/10.1590/1982-3703003392015

How to cite: Moraes, T. D., Zambroni-de-Souza, P. C., \& Schwartz, Y. (2017). Usages of simulators during professional training for driving road transportation. Psicologia: Ciência e Profissão, 37(1): 192-207. https://doi.org/10.1590/1982-3703003392015

Cómo citar: Moraes, T. D., Zambroni-de-Souza, P. C., \& Schwartz, Y. (2017). Usos de simuladores durante la formación profesional para la conducción de vehículos de transporte terrestre por carretera. Psicologia: Ciência e Profissão, 37(1): 192-207. https://doi.org/ 10.1590/1982-3703003392015 